\title{
Differential microRNA expression in renal cell carcinoma
}

\author{
TINGTING CHENG ${ }^{1}$, LINA WANG $^{1}$, YUYAO LI $^{1}$, CHEN HUANG $^{2}$, LINGXIA ZENG $^{3}$ and JIN YANG ${ }^{1}$ \\ ${ }^{1}$ Department of Medical Oncology, First Affiliated Hospital of Medical School; \\ ${ }^{2}$ Department of Genetics and Molecular Biology; ${ }^{3}$ Department of Statistics, \\ Medical School, Xi'an Jiaotong University, Xi'an, Shaanxi 710061, P.R. China
}

Received December 22, 2012; Accepted May 14, 2013

DOI: $10.3892 / 01.2013 .1460$

\begin{abstract}
The present study aimed to detect microRNA expression levels in the tissues and sera of patients with clear cell renal cell carcinoma (ccRCC). The association of microRNA expression with ccRCC clinical pathology was analyzed, and the potential of the microRNAs as ccRCC serum markers and the significance of their expression in the clinical diagnosis, staging, prognosis and selection of new therapeutic targets for ccRCC were discussed. Specific microRNAs were selected according to the associated literature. TaqMan quantitative polymerase chain reaction (qPCR) technology was used to determine the expression levels of selected microRNAs. miR-34a, miR-224 and miR-21 were upregulated, whereas miR-141, miR-149 and miR-429 were downregulated in the ccRCC tissues $(\mathrm{P}<0.01)$. The expression of miR-221 and miR-211 was not significant in the ccRCC tissues $(\mathrm{P}>0.05)$. miR-34a, miR-21 and miR-224 were upregulated and miR-141 was downregulated in the sera of patients with ccRCC $(\mathrm{P}<0.01)$, while the expression of miR-149 and miR-429 was not significant $(\mathrm{P}>0.05)$. The serum miR-21 expression levels were significantly correlated with the clinical staging of the patients with ccRCC $(\mathrm{P}<0.05)$. miR-34a, miR-21 and miR-224 are upregulated in the tissues and sera of patients with ccRCC, whereas miR-141 is downregulated. miR-21 and miR-141 are associated with ccRCC and are, thus, potential ccRCC serum markers.
\end{abstract}

\section{Introduction}

Renal cell carcinoma (RCC) accounts for 2-3\% of adult malignancies and clear cell RCC (ccRCC) accounts for 80-90\% of all RCCs (1). In recent years, the incidence of ccRCC has evidently increased and its mortality rate has reached $40 \%$ (2). Among ccRCC patients, $\sim 40 \%$ are in the late stage at diagnosis due to the lack of early-stage diagnostic markers. Up to $30-40 \%$ of patients with early-stage local ccRCC suffer from

Correspondence to: Professor Jin Yang, Department of Medical Oncology, First Affiliated Hospital of Medical School, Xi'an Jiaotong University, No. 277 Yanta West Road, Xi'an, Shanxi 710061, P.R. China

E-mail: jinyangcn@126.com

Key words: microRNA, renal cell carcinoma, radical nephrectomy relapse or metastasis, even after radical nephrectomy. ccRCC is insensitive to chemotherapy and radiotherapy; therefore, effective post-operative adjuvant therapies are lacking (3). The combined traditional immune therapy using interleukin (IL)-II and interferon does not improve the survival of patients with relapsed or advanced ccRCC (4), with five- and 10-year survival rates of $<10 \%$ (5). At present, molecular-targeted therapy has been used in post-operative adjuvant therapy for patients with early-stage ccRCC or as palliative treatment for patients with advanced ccRCC (6-12). Although the objective effective rate has evidently increased, the overall survival rate among ccRCC patients is low.

The early stages of ccRCC are clinically asymptomatic. Although the advanced stages of ccRCC may have intermittent hematuria, lumbago and abdominal masses, the occurrence rate of these symptoms does not reach $15 \%$. The patients are usually only diagnosed in the advanced stage when these symptoms are observed and the tumor has already extensively progressed and distantly metastasized. The prognosis of these patients is poor (13). Therefore, searching for tumor markers, which may be used for the early diagnosis, follow-up and clinical treatment of ccRCC patients, has become a focus of basic and clinical ccRCC research.

MicroRNAs do not encode proteins. Instead, they promote the degradation of targeted mRNA by being completely complementary to the mRNA or inhibiting the expression of the targeted mRNA by being partially complementary. MicroRNAs are involved in gene regulation at the translation level and are present in tissues, as well as in sera (14). Studies have shown that microRNAs function as oncogenes or cancer suppressor genes. They regulate the normal growth and development of organisms and are involved in tumor formation and development via the mediation of downstream target genes. MicroRNAs also function in tumor cell proliferation, apoptosis, invasion and tumor vessel formation. Studies have shown that microRNAs have specific expression profiles in the tissues and sera of patients with renal cancer (15-24). The tumor tissue may be distinguished from the adjacent normal tissue and thus patients with ccRCC may be distinguished from healthy individuals. However, no tests have confirmed the consistency of microRNA expression in the tissues and sera of patients with ccRCC. Therefore, the use of microRNA as a diagnostic marker for ccRCC is significant.

The TaqMan quantitative polymerase chain reaction (qPCR) was used in the present study to determine the microRNA 
expression levels in the tumor and normal tissues of patients with ccRCC and in the serum of patients with ccRCC or benign kidney lesions (BKL). The association between differential microRNA expression and gender, age, clinical stage, tumor size, invasive condition of the renal capsule and pathological classification of patients with ccRCC was also studied.

\section{Materials and methods}

Materials. In total, 30 pairs of ccRCC tumor tissues and normal tissue samples, 12 pre-operative serum samples from patients with ccRCC and 12 serum samples from patients with BKL were collected between April 2010 and March 2011 at the Department of Urological Surgery, First Hospital of Xi'an Jiaotong University (Xi'an, Shaanxi, China). All patients who donated tissue and ccRCC serum samples were diagnosed with ccRCC via post-operative pathology. None of these patients received pre-operative irradiation or chemotherapy.

Of the 30 tissue samples from the patients with ccRCC, 15 were from males and 15 were from females, with a mean patient age of 55 years (range, 19-74 years). Of these 30 patients, 17 were in clinical stage I and 13 were in stage II. One patient was of pathological class I, 21 were class II and eight were class III. The tumor sizes were $\leq 7 \mathrm{~cm}$ in 21 patients and $>7 \mathrm{~cm}$ in the remaining nine patients. A total of 26 patients had invasion of the renal capsule, whereas four did not.

Of the 12 serum samples from the patients with ccRCC, eight were from males and four were from females, with a mean patient age of 54 years (range, 30-83 years). Of these 12 patients, nine were in clinical stage I, two were in stage II and one was in stage III. Three patients were of pathological class I, seven were class II, one was class III and one was class IV. The tumors were $\leq 7 \mathrm{~cm}$ in size in nine patients and $>7 \mathrm{~cm}$ in the remaining three. Ten patients had invasion of the renal capsule, whereas two did not. The present study was conducted in accordance with the declaration of Helsinki and with approval from the Ethics Committee of Xi'an Jiaotong University. Written informed consent was obtained from all participants.

Methods. MicroRNAs were selected for the study based on reviewed literature. To be selected, the microRNA must have been associated with ccRCC and reported in at least two studies. miR-34a, miR-21, miR-224, miR-141, miR-149, miR-429, miR-221 and miR-211 were finally selected. Total RNA was extracted from the tissue (RNAfast 200, Biotechnology Co., Ltd., Shanghai, China) and serum (mirVana ${ }^{\mathrm{TM}}$ PARIS kit, Applied Biosystems Inc., Foster City, CA, USA) samples according to the instructions of the respective extraction kits. The RNA was reverse transcribed into cDNA using the additional poly(A) tail reaction method according to the instructions of the reverse transcription kit (PrimeScript ${ }^{\circledR}$ One-Step RT-PCR kit, Takara Biomedical Technology, Dalian, China). The qPCR reaction was performed according to the instructions of the qPCR kit (SYBR ${ }^{\circledR}$ Premix Ex Taq ${ }^{\mathrm{TM}}$ II, Takara Biomedical Technology).

Data analysis. Relative quantification was selected to determine the microRNA amplification. The changes in microRNA amplification were normalized with the housekeeping gene, U6. The fold changes in the microRNA of the results of the qPCR were calculated for each sample using $2^{-\Delta \Delta C t}$, where $\Delta \Delta \mathrm{CT}_{1}=\left(\mathrm{CT}_{\mathrm{miR}}-\mathrm{CT}_{\mathrm{U} 6}\right)_{\text {tumor }}-\left(\mathrm{CT}_{\mathrm{miR}}-\mathrm{CT}_{\mathrm{U} 6}\right)_{\text {adjacent }}$ in the tissue samples and $\Delta \Delta \mathrm{CT}_{2}=\left(\mathrm{CT}_{\mathrm{miR}}-\mathrm{CT}_{\mathrm{U} 6}\right)_{\mathrm{ccRCC}}-\left(\mathrm{CT}_{\mathrm{miR}}-\mathrm{CT}_{\mathrm{U} 6}\right)_{\mathrm{BKL}}$ in the serum samples. A change in $2^{-\Delta \Delta \mathrm{Ct}}$ of $>1.5$ was considered an amplification or reduction of the microRNAs.

Statistical analysis. All statistical analyses were performed using SPSS 13.0 software (SPSS Inc., Chicago, IL, USA). The differences between the tumor and adjacent tissues were tested using the paired samples t-test. The differences between the serum of patients with ccRCC and BKL were tested using an independent samples t-test or non-parametric test. The clinical correlation analysis was performed using the variance test or Kruskal-Wallis test. $\mathrm{P}<0.05$ was considered to indicate a statistically significant difference.

\section{Results}

Tissue results. As shown in Fig. 1, of the 30 tissue samples, 21 expressed miR-34a, 23 expressed miR-21 and 24 expressed miR-224. The expression was upregulated in the tumor tissues compared with the normal adjacent tissues $(\mathrm{P}<0.01$ for all three microRNAs). The fold changes for miR-34a, miR-21 and miR-224 were 6.2, 22.5 and 27.5, respectively.

As shown in Fig. 2, of the 30 tissue samples, 26 expressed miR-141, 21 expressed miR-149 and 22 expressed miR-429. The expression was downregulated in the tumor tissues compared with the normal adjacent tissues $(\mathrm{P}<0.01$ for all three microRNAs). The fold changes for miR-141, miR-149 and miR-429 were 6.3, 1.7 and 2.4, respectively.

As shown in Fig. 3, no significant differences were observed in the expression of miR-211 and miR-221 in the tumor and normal adjacent tissues $(\mathrm{P}=0.339$ and $\mathrm{P}=0.271$, respectively). Of the 30 tissue samples, miR-211 expression was upregulated in seven and downregulated in 18, whereas miR-221 was upregulated in eight and downregulated in seven.

Serum results. As shown in Fig. 4, of the 12 serum samples, 11 expressed miR-34a, 12 expressed miR-21 and nine expressed miR-224. The microRNA expression was upregulated in the serum of patients with ccRCC compared with those with BKL ( $\mathrm{P}<0.01$ for all three microRNAs). The fold changes for miR-34a, miR-21 and miR-224 were 84, 277 and 4, respectively.

As shown in Fig. 5, all 12 serum samples expressed miR-141. The expression was downregulated in the serum of patients with ccRCC compared with those with BKL $(\mathrm{P}<0.01)$. The fold change for miR-141 was $5.8 \times 10^{7}$.

As shown in Fig. 6, no significant differences were observed in the expression of miR-149 and miR-429 in the serum of patients with ccRCC and $\mathrm{BKL}(\mathrm{P}=0.754$ and $\mathrm{P}=0.657$, respectively).

Correlation between microRNA expression and clinicopathological characteristics in ccRCC patients. The correlations between microRNA expression in the 30 tissue samples from the ccRCC patients and the clinicopathological features are presented in Table I. No significant correlations were observed 


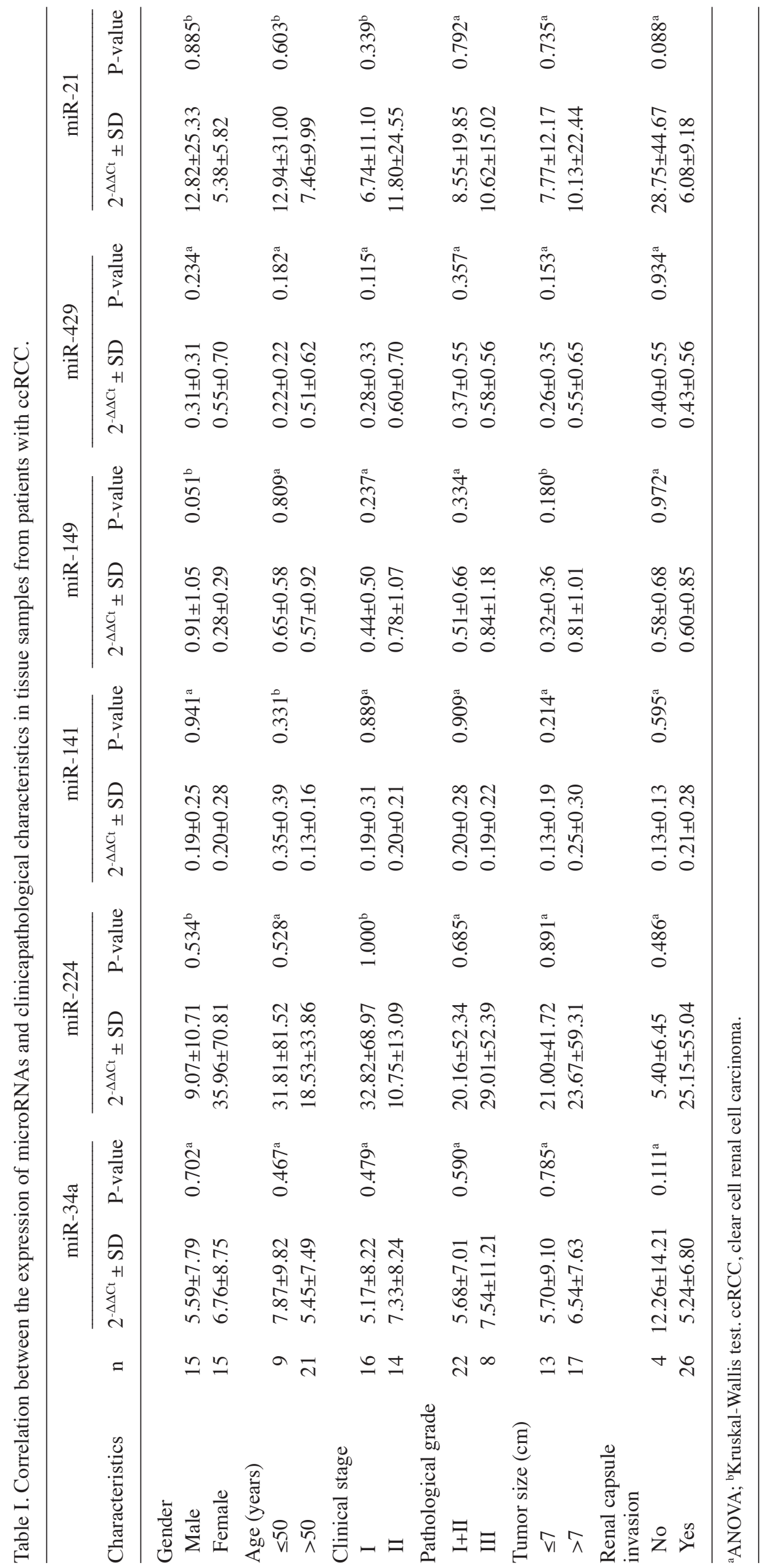




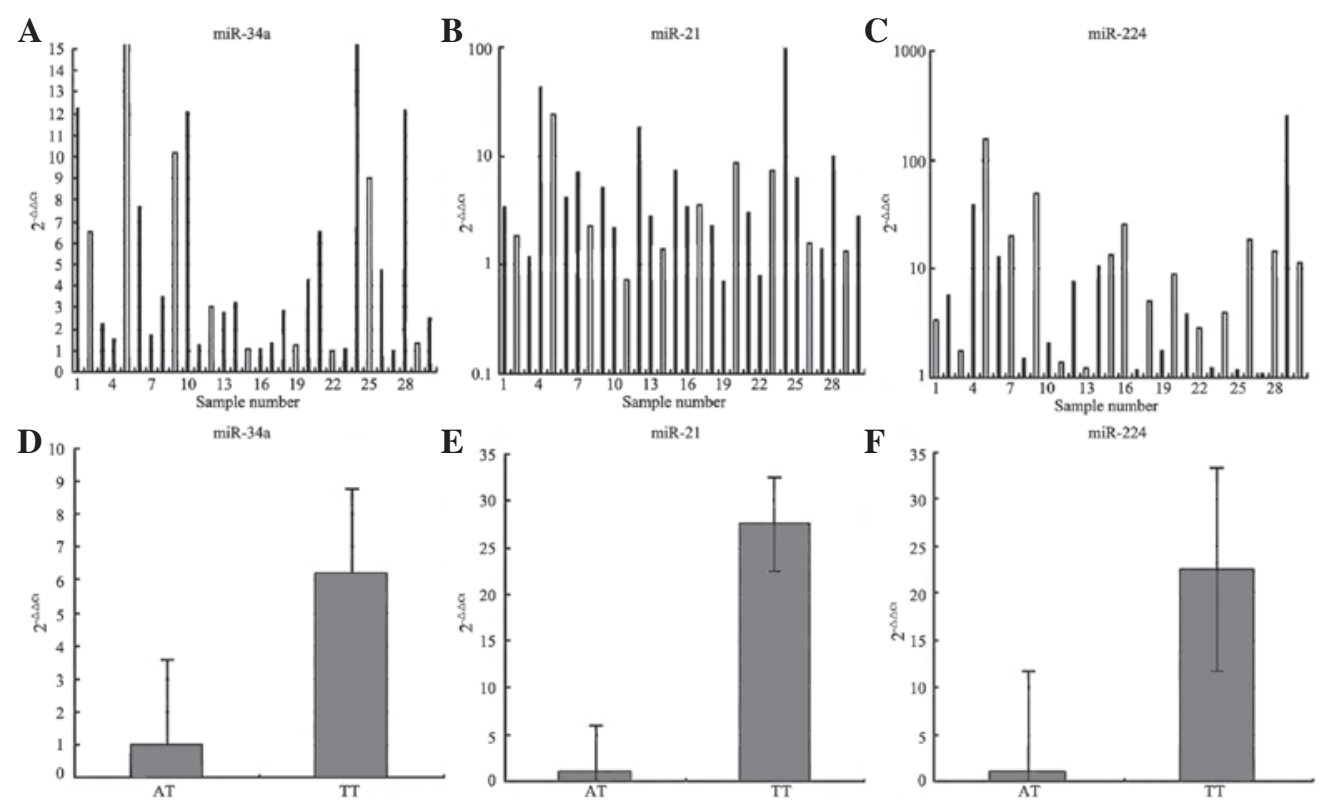

Figure 1. Individual (A, B, C) and general (D, E, F) relative quantification for miR-34a, miR-21 and miR-224 in tissue samples from patients with clear cell renal cell carcinoma (ccRCC). AT, adjacent tissue; TT, tumor tissue.
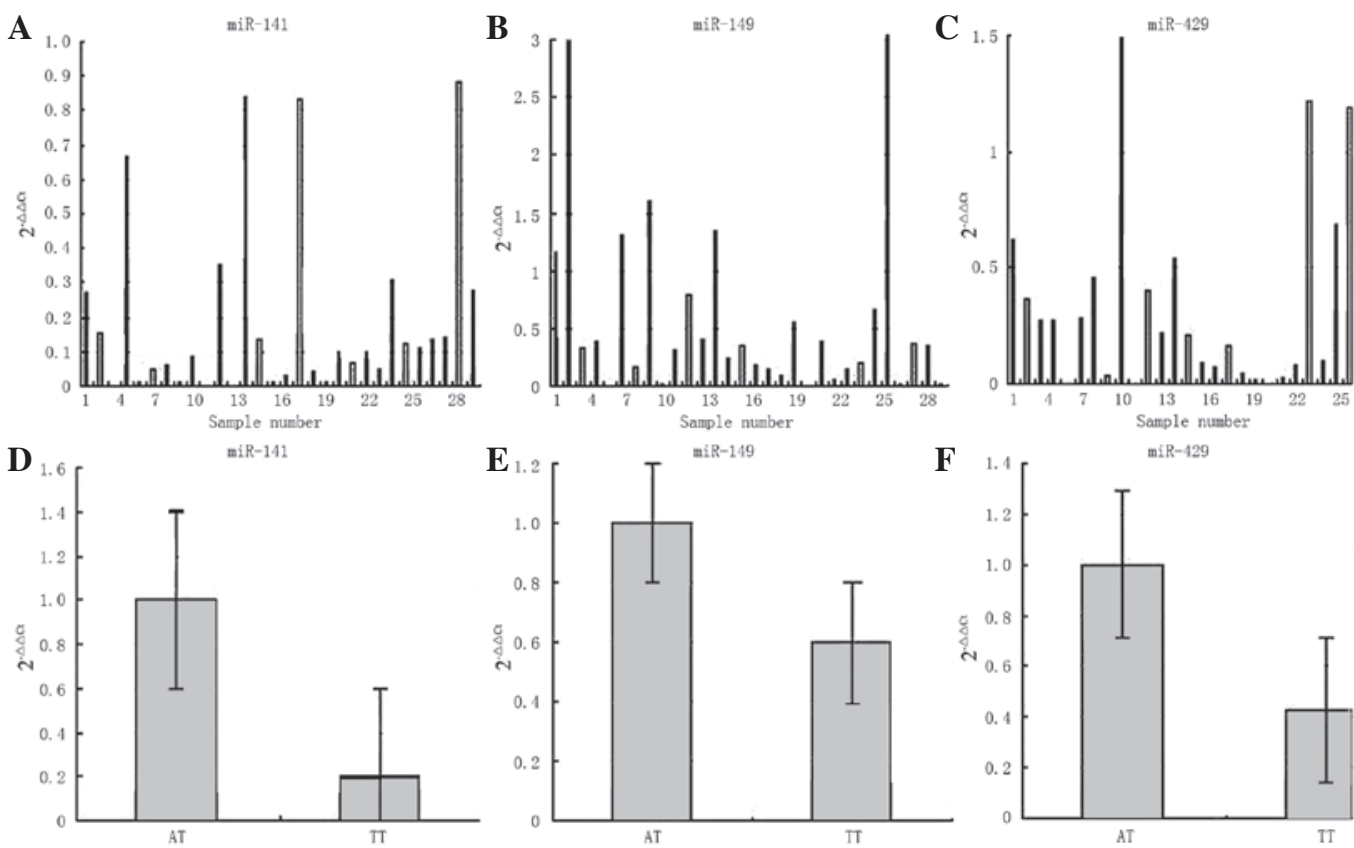

Figure 2. Individual (A, B, C) and general (D, E, F) relative quantification for miR-141, miR-149 and miR-429 in tissue samples from patients with clear cell renal cell carcinoma (ccRCC). AT, adjacent tissue; TT, tumor tissue.

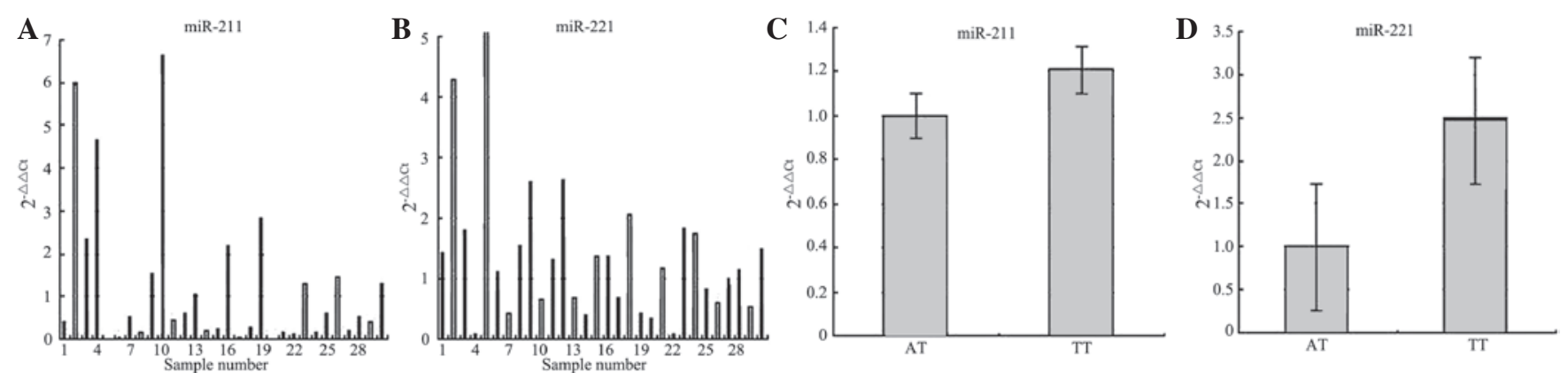

Figure 3. Individual (A, B) and general (C, D) relative quantification for miR-211 and miR-221 in tissue samples from patients with clear cell renal cell carcinoma (ccRCC). AT, adjacent tissue; TT, tumor tissue. 

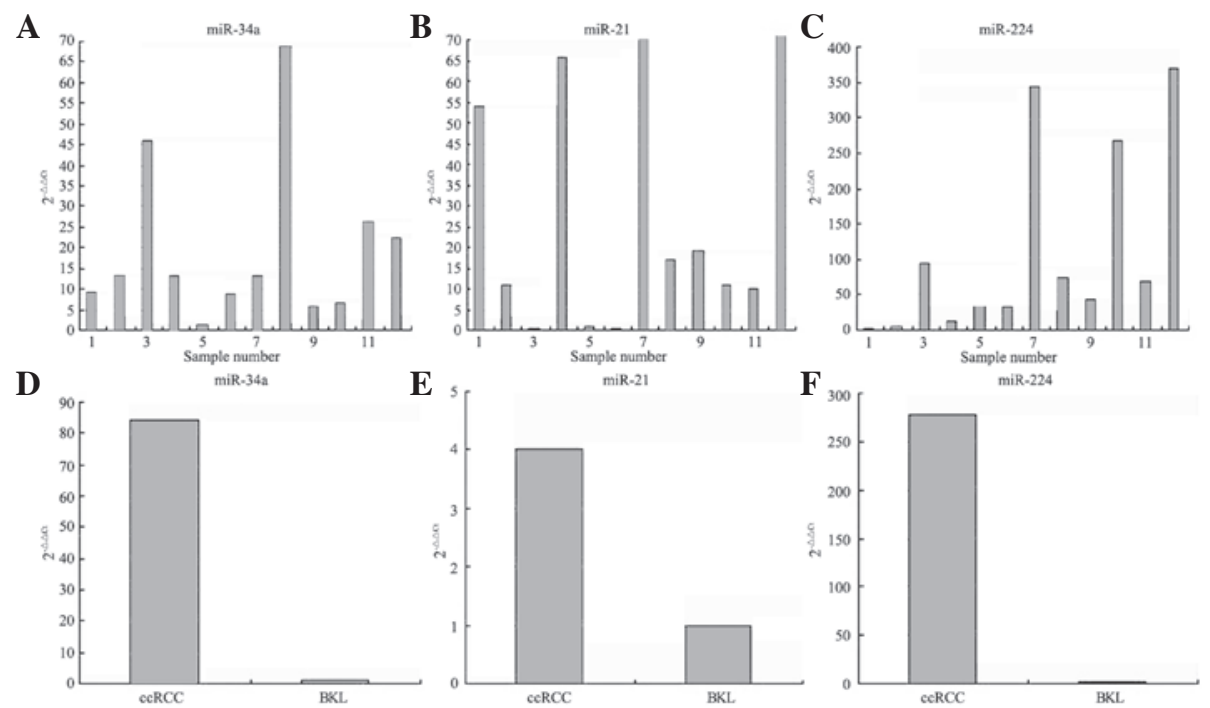

Figure 4. Individual (A, B, C) and general (D, E, F) relative quantification for miR-34a, miR-21 and miR-224 in serum samples from patients with ccRCC compared with those with BKL. ccRCC, clear cell renal cell carcinoma; BKL, benign kidney lesion.
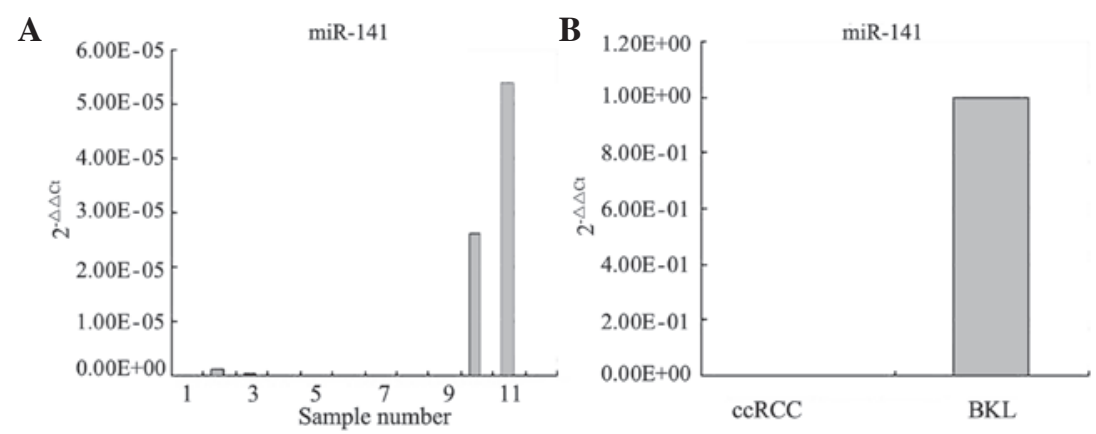

Figure 5. Individual (A) and general (B) relative quantification for miR-141 in serum samples from patients with ccRCC compared with those with BKL. ccRCC, clear cell renal cell carcinoma; BKL, benign kidney lesion.
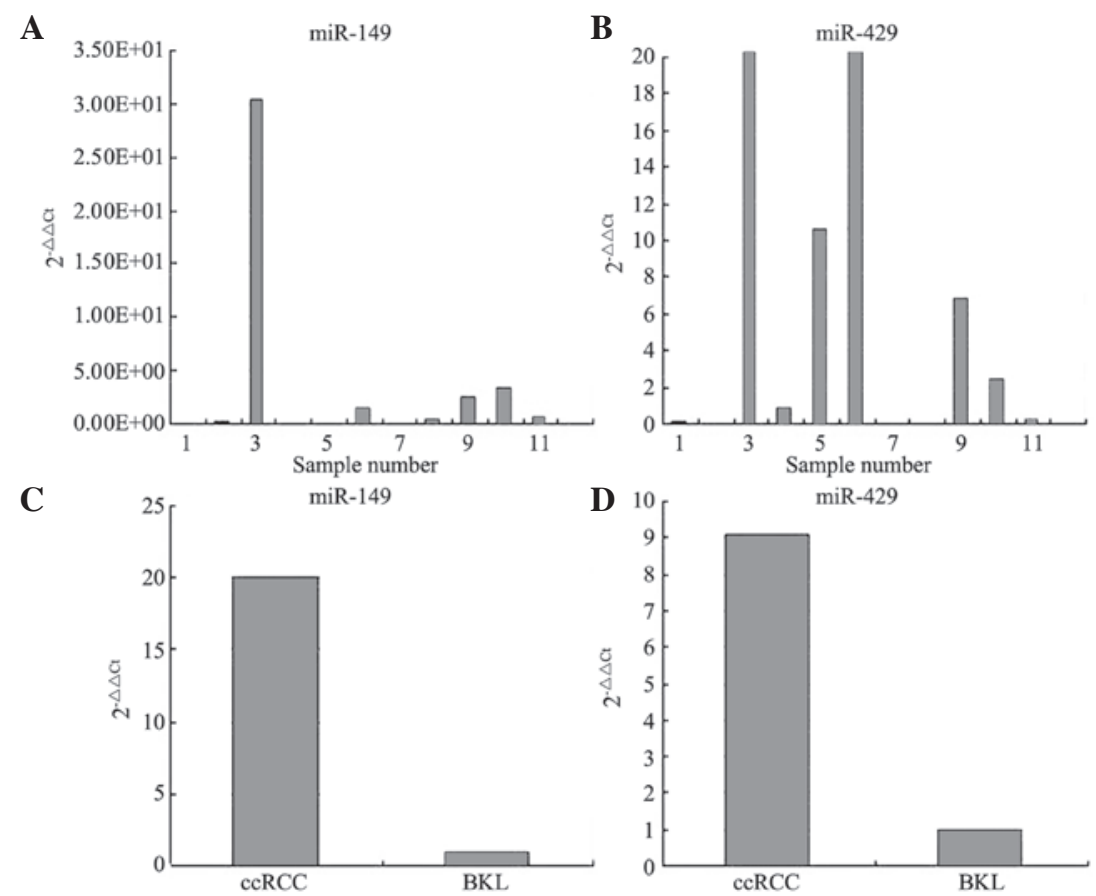

Figure 6. Individual (A, B) and general (C, D) relative quantification for miR-149 and miR-429 in serum samples from patients with ccRCC compared with those with BKL. ccRCC, clear cell renal cell carcinoma; BKL, benign kidney lesion. 


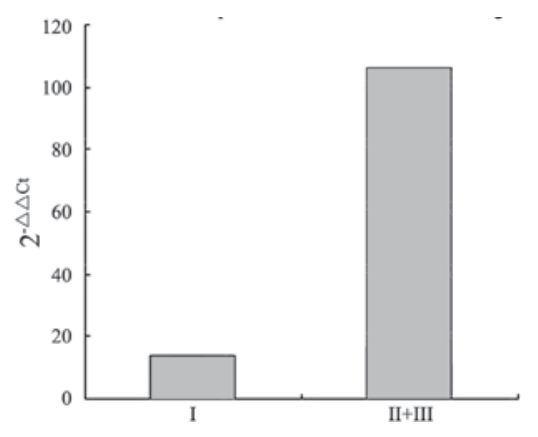

Figure 7. Correlation between miR-21 and clinical stage in serum samples of clear cell renal cell carcinoma patients $(\mathrm{P}<0.05)$

between microRNA expression and gender, age, clinical stage, pathological grade, tumor size or invasive condition of the renal capsule of tissue samples from patients with ccRCC.

The correlation between microRNA expression in the 12 serum samples from patients with ccRCC and the clinicopathological features is shown in Table II. The correlation between miR-21 expression and the clinical stage was significant $(\mathrm{P}<0.05$; Fig. 7). The correlation between miR-224 expression and gender was significant $(\mathrm{P}<0.05$; Fig. 7) No significant correlation was observed between the expression of the other microRNAs and gender, age, clinical stage, pathological grade, tumor size and invasive condition of renal capsule of the serum samples from the patients with ccRCC.

\section{Discussion}

As a solid tumor, ccRCC is resistant to chemotherapy and radiotherapy. The main therapeutic method for local ccRCC is radical nephrectomy, which has a five-year survival rate of $60-80 \%$. However, the five-year survival rate of patients with metastatic ccRCC is $<10 \%$ due to the lack of effective post-operative adjuvant therapy. The early stage of ccRCC is clinically asymptomatic; thus, the majority of patients with ccRCC already have distant metastasis with clinical symptoms upon diagnosis. At present, no tumor marker for early ccRCC diagnosis has been identified. Therefore, the present study attempted to identify a tumor marker that may be used to diagnose the early stage of ccRCC based on the quantity of microRNA expression in the tumors and sera of ccRCC patients.

The study showed that the expression levels of miR-34a, miR-224 and miR-21 were significantly higher in ccRCC tumor tissues compared with the adjacent tissues $(\mathrm{P}<0.01)$. The expression levels of miR-141, miR-149 and miR-429 were significantly lower in the tumor tissues compared with the adjacent tissues $(\mathrm{P}<0.01)$. The results were consistent with those of previous studies (15-24). However, the microRNA expression levels had no correlation with the clinical data of the patients with ccRCC in the present study. Moreover, the expression levels of miR-34a, miR-224 and miR-21 were significantly higher in the sera of the patients with ccRCC compared with those with BKL $(\mathrm{P}<0.01)$, while the expression levels of miR-141 were significantly lower in the sera of the ccRCC patients compared with those with BKL $(\mathrm{P}<0.01)$. The expression profiles of miR-34a, miR-224, miR-21 and
miR-141 in the sera of patients with ccRCC were consistent with those of the corresponding tumor tissue samples. The miR-21 expression levels in the serum of the patients with ccRCC were correlated with the patients' clinical stage $(\mathrm{P}<0.05)$ and miR-224 expression levels were correlated with gender The present study showed that miR-34a, miR-224, miR-21 and miR-141 are potential ccRCC tumor markers. The study demonstrated that the expression of miR-224 and miR-141 in the sera of patients with ccRCC was significantly different. However, no significant correlation was observed between microRNA expression and the clinicopathological characteristics of the patients, with the exception of the significant correlation between serum miR-21 expression and ccRCC clinical stage due to the smaller sample size. Further studies with greater sample sizes are required to confirm microRNA as a ccRCC serum marker.

Studies on ccRCC have demonstrated that microRNA expression differs significantly between ccRCC tumors and the adjacent normal tissues (15-24). In 2010, Chen (15) demonstrated that microRNA expression was significantly different in the ccRCC serum. However, the consistency of microRNA expression in the tumor tissues and sera of ccRCC patients has not yet been demonstrated. The present study is the first to demonstrate the significance of the expression of miR-34a, miR-224, miR-21 and miR-141 in the tissues and sera of patients with ccRCC, with coinciding expression trends.

In 2008, Chen et al (14) demonstrated that microRNAs are resistant to RNase, indicating that they are maintained in the serum and plasma of humans and other animals. Serum microRNAs are normally derived from circulatory hemocytes, whereas tumor-related microRNAs are derived from circulatory hemocytes, as well as tumor tissues and tumor-affected tissues. Moreover, Mitchell et al (25) further demonstrated that microRNAs from tumor cells enter the blood circulation and that microRNA expression levels are correlated with tumor size. The microRNAs result from the initiating tumor cell secretions and are potential tumor serum markers. Wei et al (26) and Tomimaru et al (27) considered that miR-21 may be used as a tumor serum marker to diagnose hepatocellular carcinoma and non-small cell lung cancer. Certain studies have shown that miR-141 has the potential as a tumor serum marker for prostatic and colorectal cancer (28-31).

Inhibiting miR-21 expression arrests the cell cycle of tumor cells and induces apoptosis in the ccRCC cell line. Consequently, the tumor cell invasiveness and metastatic capacity would also decline. The results of a study by Zaman et al also showed that the average survival time of ccRCC patients with low miR-21 expression was more than five years and that the five-year survival rate of ccRCC patients with high miR-21 expression was only $50 \%$. In addition, the miR-21 expression levels increased with the increasing ccRCC pathological grade (32).

Another study showed that miR-224 expression in ccRCC tumor tissues was four-fold higher compared with a control group $(\mathrm{P}<0.01)$ (33). The study suggested that miR-224 acts directly on the DIO1 UTR in ccRCC. The specific change in the tumors is negatively correlated with the concentration of intracellular DIO1 and T3. Induced miR-224 expression in Caki-2 cells causes a significant decline in DIO1 mRNA levels $(\mathrm{P}<0.01)(33)$. 


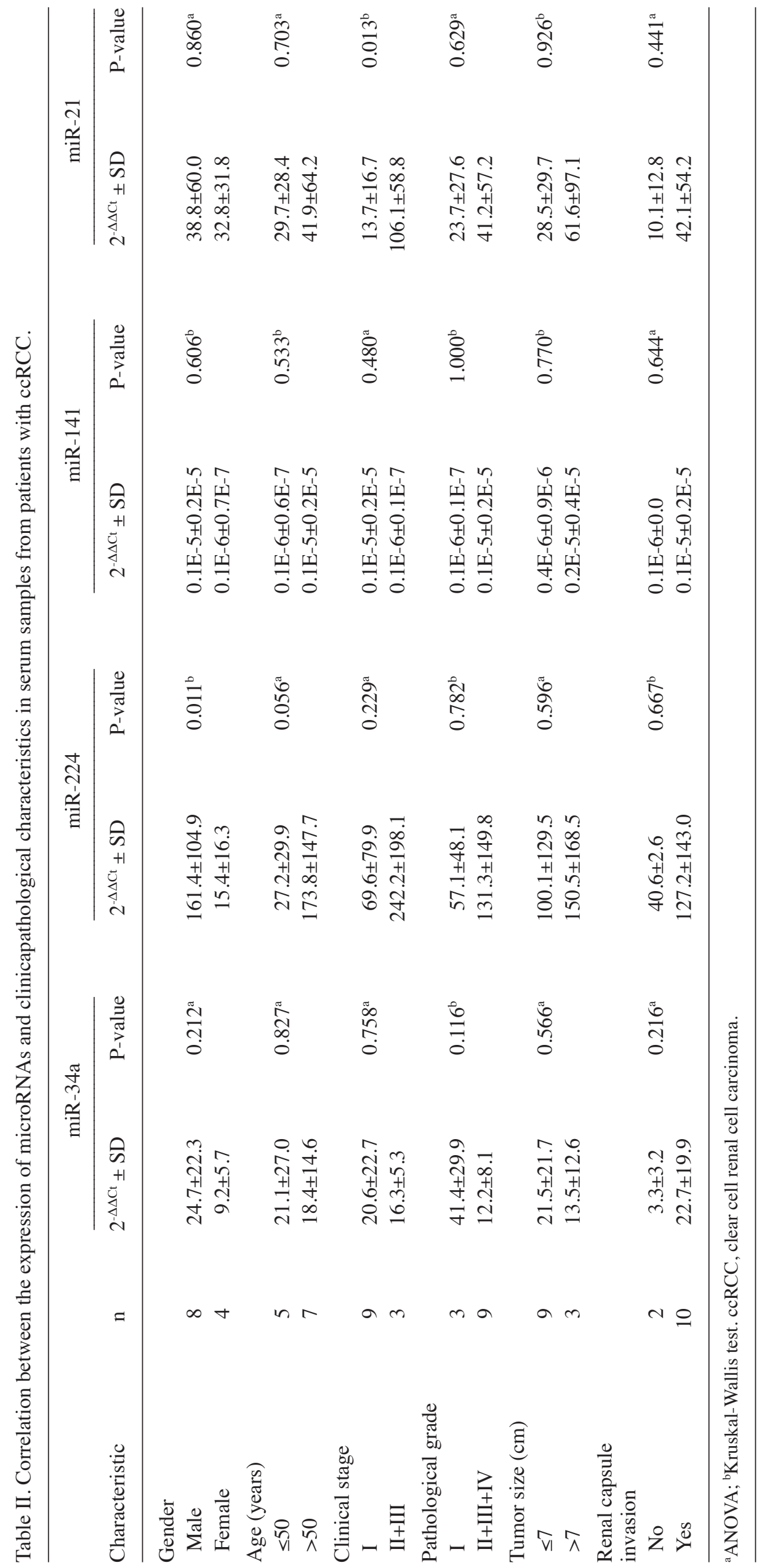


miR-141 expression is downregulated significantly in ccRCC, which is associated with the epithelial-mesenchymal transition (EMT) (34). EMT causes the loss of cell adhesion and promotes cancer cell metastasis, including ccRCC metastasis. Liu et al (21) suggested that the loss of expression of miR-149, miR-200c and miR-141 causes the activation of oncogenes [KCNMA1, LOX, vascular endothelial growth factor A (VEGFA) and SEMA6A]. The expression of the miR-200 family (miR-200a*, miR-200b, miR-200c and miR-141) was negatively correlated with VEGFA, and SEMA6A was the direct target gene of miR-141.

miR-141 and miR-21 are involved in the progression of ccRCC. In the present study, no significant correlation was observed between the other microRNAs and the ccRCC clinicopathological data in the tissue and serum from patients with ccRCC. However, serum miR-21 expression was significantly correlated with the clinical staging of ccRCC and serum miR-224 expression was significantly correlated with gender due to the small sample size. Therefore, miR-141, miR-224 and miR-21 are potential ccRCC serum markers.

\section{Acknowledgements}

The present study was supported by the Department of Genetics and Molecular Biology and Statistics, Medical School, Xi'an Jiaotong University, Xi'an, Shaanxi, China. The authors would like to thank Professors Yang Jin, Huang Chen and Zeng Lingxia for their help and support. This study was supported by the National Natural Science Foundation of China (No. 81172171). The molecular mechanism of abnormal expression and potential targeted therapy value of Angiomotin in clear cell renal cell carcinoma

\section{References}

1. Jemal A, Siegel R, Ward E, et al: Cancer statistics, 2006. CA Cancer J Clin 56: 106-130, 2006.

2. Gottardo F, Liu CG, Ferracin M, et al: Micro-RNA profiling in kidney and bladder cancers. Urol Oncol 25: 387-392, 2007.

3. Escudier B: Advanced renal cell carcinoma: current and emerging management strategies. Drugs 67: 1257-1264, 2007.

4. Broghammer EL and Ratliff TL: Immunotherapy of urologic tumors: principles and progress. Urol Oncol 7: 45-56, 2002.

5. Mulders P, Figlin R, deKernion JB, et al: Renal cell carcinoma: recent progress and future directions. Cancer Res 57: 5189-5195, 1997.

6. Bukowski RM, Eisen T, Szczylik C, et al: Final results of the randomized phase III trial of sorafenib in advanced renal cell carcinoma: Survival and biomarker analysis. J Clin Oncol 25: $5023,2007$.

7. Motzer RJ, Hutson TE, Tomczak P, et al: Overall survival and updated results for sunitinib compared with interferon alfa in patients with metastatic renal cell carcinoma. J Clin Oncol 27: 3584-3590, 2009.

8. Hudes G, Carducci M, Tomczak P, et al: Temsirolimus, interferon alfa, or both for advanced renal-cell carcinoma. N Engl J Med 356: 2271-2281, 2007.

9. Escudier B, Pluzanska A, Koralewski P, et al: Bevacizumab plus interferon alfa-2a for treatment of metastatic renal cell carcinoma: a randomised, double-blind phase III trial. Lancet 370: 2103-2111, 2007.

10. Jonasch E, Wood CG, Matin SF, et al: Phase II presurgical feasibility study of bevacizumab in untreated patients with metastatic renal cell carcinoma. J Clin Oncol 27: 4076-4081, 2009.

11. Sternberg CN, Davis ID, Mardial J, et al: Pazopanib in locally advanced or metastatic renal cell carcinoma: results of a randomised phase III trial. J Clin Oncol 28: 1061-1068, 2010.
12. Motzer RJ, Escudier B, Oudard S, et al: Phase 3 trial of everolimus for metastatic renal cell carcinoma: final results and analysis of prognostic factors. Cancer 116: 4256-4265, 2010.

13. Walsh PC, Retik AB, Vaugh ED, et al (eds): Campbell's Urology. 8th edition. WB Saunders Company, Philadelphia, PA, pp2672-2719, 2002.

14. Chen X, Ba Y, Ma L, et al: Characterization of microRNAs in serum: a novel class of biomarkers for diagnosis of cancer and other disease. Cell Res 18: 997-1006, 2008.

15. Chen ZY: The expression of circulated microRNA in ccRCC (unpublished $\mathrm{PhD}$ thesis). Zhongnan University, 2010.

16. Nakada C, Matsuura K, Tsukamoto Y, et al: Genome-wide microRNA expression profiling in renal cell carcinoma: significant down-regulation of miR-141 and miR-200c. J Pathol 216: 418-427, 2008.

17. Jung M, Mollenkopf HJ, Grimm C, et al: MicroRNA profiling of clear cell renal cell cancer identifies a robust signature to define renal malignancy. J Cell Mol Med 13: 3918-3928, 2009.

18. Li M: MicroRNA expression profiling in renal cell carcinoma and its potential clinical significance (unpublished $\mathrm{PhD}$ thesis). Zhongshan University, 2009.

19. Huang Y, Dai Y, Yang J, et al: Microarray analysis of microRNA expression in renal clear cell carcinoma. Eur J Surg Oncol 35: $1119-1123,2009$.

20. Slaby O, Jancovicov J, Lakomy R, et al: Expression of miRNA-106b in conventional renal cell carcinoma is a potential marker for prediction of early metastasis after nephrectomy. J Exp Clin Cancer Res 29: 90, 2010.

21. Liu H, Brannon AR, Reddy AR, et al: Identifying mRNA targets of microRNA dysregulated in cancer: with application to clear cell Renal Cell Carcinoma. BMC Syst Biol 4: 51, 2010.

22. Juan D, Alexe G, Antes $T$, et al: Identification of a microRNA panel for clear-cell kidney cancer. Urology 75: 835-841, 2010.

23. Chow TF, Youssef YM, Lianidou E, et al: Differential expression profiling of microRNAs and their potential involvement in renal cell carcinoma pathogenesis. Clin Biochem 43: 150-158, 2010.

24. Yi Z, Fu Y, Zhao S, Zhang X and Ma C: Differential expression of miRNA patterns in renal cell carcinoma and nontumorous tissues. J Cancer Res Clin Oncol 136: 855-862, 2010.

25. Mitchell PS, Parkin RK, Kroh EM, et al: Circulating microRNAs as stable blood-based markers for cancer detection. Proc Natl Acad Sci USA 105: 10513-10518, 2008.

26. Wei J, Gao W, Zhu CJ, et al: Identification of plasma microRNA-21 as a biomarker for early detection and chemosensitivity of non-small cell lung cancer. Chin J Cancer 30: 407-414, 2011.

27. Tomimaru Y, Equchi H, Naqano $\mathrm{H}$, et al: Circulating microRNA-21 as a novel biomarker for hepatocellular carcinoma. J Hepatol 56: 167-175, 2012.

28. Nam EJ, Yoon H, Kim SW, et al: MicroRNA expression profiles in serous ovarian carcinoma. Clin Cancer Res 14: 2690-2695, 2008.

29. Gonzales JC, Fink LM, Goodman OB Jr, Symanowski JT, Vogelzang NJ and Ward DC: Comparison of circulating MicroRNA 141 to circulating tumor cells, lactate dehydrogenase, and prostate- specific antigen for determining treatment response in patients with metastatic prostate cancer. Clin Genitourin Cancer 9: 39-45, 2011.

30. Yaman Agaoglu F, Kovancilar M, Dizdar Y, et al: Investigation of miR-21, miR-141, miR-221 in blood circulation of patients with prostate cancer. Tumour Biol 32: 583-588, 2011.

31. Cheng H, Zhang L, Cogdell DE, et al: Circulating plasma MiR-141 is a novel biomarker for metastatic colon cancer and predicts poor prognosis. PloS One 6: e17745, 2011.

32. Zaman MS, Shahryari V, Deng G, et al: Up-regulation of microRNA-21 correlates with lower kidney cancer survival. PLoS One 7: e31060, 2012.

33. Boquslawska J, Wojcicka A, Piekielko-Witkowska A, Master A and Nauman A: MiR-224 targets the 3'UTR of type 1 5'-iodothyronine deiodinase possibly contributing to tissue hypothyroidism in renal cancer. PLoS One 6: e24541, 2011.

34. Gibbons DL, Lin W, Creighton CJ, et al: Contextual extracellular cues promote tumor cell EMT and metastasis by regulating miR-200 family expression. Genes Dev 23: 2140-2151, 2009. 